VNU Journal of Science: Natural Sciences and Technology

Journal homepage: https://js.vnu.edu.vn/NST

Original Article

\title{
Postbuckling Behavior of Functionally Graded Multilayer Graphene Nanocomposite Plate under Mechanical and Thermal Loads on Elastic Foundations
}

\author{
Pham Hong Cong ${ }^{1}$, Nguyen Dinh Duc ${ }^{2, *}$ \\ ${ }^{1}$ Centre for Informatics and Computing (CIC), Vietnam Academy of Science and Technology, \\ 18 Hoang Quoc Viet, Cau Giay, Hanoi, Vietnam \\ ${ }^{2}$ Advanced Materials and Structures Laboratory, VNU University of Engineering and Technology (UET), \\ 144 Xuan Thuy, Cau Giay, Hanoi, Vietnam \\ Received 08 November 2019 \\ Revised 03 December 2019; Accepted 03 December 2019
}

\begin{abstract}
This paper presents an analytical approach to postbuckling behaviors of functionally graded multilayer nanocomposite plates reinforced by a low content of graphene platelets (GPLs) using the first order shear deformation theory, stress function and von Karman-type nonlinear kinematics and include the effect of an initial geometric imperfection. The weight fraction of GPL nano fillers is assumed to be constant in each individual GPL-reinforced composite (GPLRC). The modified Halpin-Tsai micromechanics model that takes into account the GPL geometry effect is adopted to estimate the effective Young's modulus of GPLRC layers. The plate is assumed to resting on Pasternak foundation model and subjected to mechanical and thermal loads. The results show the influences of the GPL distribution pattern, weight fraction, geometry, elastic foundations, mechanical and temperature loads on the postbuckling behaviors of FG multilayer GPLRC plates.
\end{abstract}

Keywords: Postbuckling; Graphene nanocomposite plate; First order shear deformation plate theory.

\section{Introduction}

Advanced materials have been considered promising reinforcement materials. To meet the demand, some smart materials are studied and created such as FGM, piezoelectric material, nanocomposite, magneto-electro material and auxetic material (negative Poisson's ratio).
Recently, a new class of promising material known as graphene has drawn considerable attention of the science and engineering communities. Graphene is a two-dimensional monolayer of sp2-bonded carbon atoms [1,2] and possesses extraordinarily material properties such as super-high mechanical strength and

\footnotetext{
${ }^{*}$ Corresponding author.

Email address: ducnd@ vnu.edu.vn

https://doi.org/10.25073/2588-1140/vnunst.4972
} 
remarkable electrical and thermal conductivities [3-5]. It was reported by researchers that the addition of a small percentage of graphene fillers in a composite could improve the composite's mechanical, electrical and thermal properties substantially [6-8].

The research on buckling and postbuckling of the functionally graded multilayer graphene nanocomposite plate and shell has been attracting considerable attention from both research and engineering. Song et al. [9, 10] studied buckling and postbuckling of biaxially compressed functionally graded multilayer graphene nanoplatelet-reinforced polymer composite plates (excluding thermal load and elastic foundation). Wu et al. [11] investigated thermal buckling and postbuckling of functionally graded graphene nanocomposite plates. Yang et al. [12] analyzed the buckling and postbuckling of functionally graded multilayer graphene platelet-reinforced composite beams. Shen et al. [13] studied the postbuckling of functionally graded graphene-reinforced composite laminated cylindrical panels under axial compression in thermal environments. Stability analysis of multifunctional advanced sandwich plates with graphene nanocomposite and porous layers was considered in [14]. Buckling and post-buckling analyses of functionally graded graphene reinforced by piezoelectric plate subjected to electric potential and axial forces were investigated in [15].

Some researches using analytical method, stress function method to study graphene structures can be mentioned [16-18]. In [16], the author considered nonlinear dynamic response and vibration of functionally graded multilayer graphene nanocomposite plate on viscoelastic Pasternak medium in thermal environment. 2D penta-graphene model was used in $[17,18]$.

From overview, it is obvious that the postbuckling of graphene plates have also attracted researchers' interests and were studied [9-11]. However, in $[9,10]$ the authors neither considered thermal load nor elastic foundation. In [11], the authors used differential quadrature
(DQ) method) but did not mention thermal load, elastic foundation and imperfect elements. In addition, in $[9,10,11]$ the stress function method was not used to the study.

Therefore, we consider postbuckling behavior of functionally graded multilayer graphene nanocomposite plate under mechanical and thermal loads and using the analytical method (stress function method, Galerkin method).

\section{Nomenclature}

$$
\begin{array}{ll}
E_{G P L}, E_{m} & \begin{array}{l}
\text { The Young's moduli of the GPL } \\
\text { and matrix, respectively. }
\end{array} \\
a_{G P L}, b_{G P L}, t_{G P L} \quad \begin{array}{l}
\text { The length, width and thickness of } \\
\text { GPL nanofillers, respectively. }
\end{array} \\
v_{G P L}, v_{m} & \begin{array}{l}
\text { The Poisson's ratios with the } \\
\text { subscripts "GPL" and "m" refering } \\
\text { to the GPL and matrix, respectively. }
\end{array} \\
\alpha_{G P L}, \alpha_{m} & \begin{array}{l}
\text { The thermal expansion coefficients } \\
\text { with the subscripts "GPL" and "m" } \\
\text { referring to the GPL and matrix } \\
\text { respectively. }
\end{array}
\end{array}
$$

\section{Functionally graded multilayer GPLRC plate model}

A rectangular laminated composite plate of length $a$, width $b$ and total thickness $h$ that is composed of a total of $N_{L}$ on Pasternak foundation model, as shown in Figure 1.

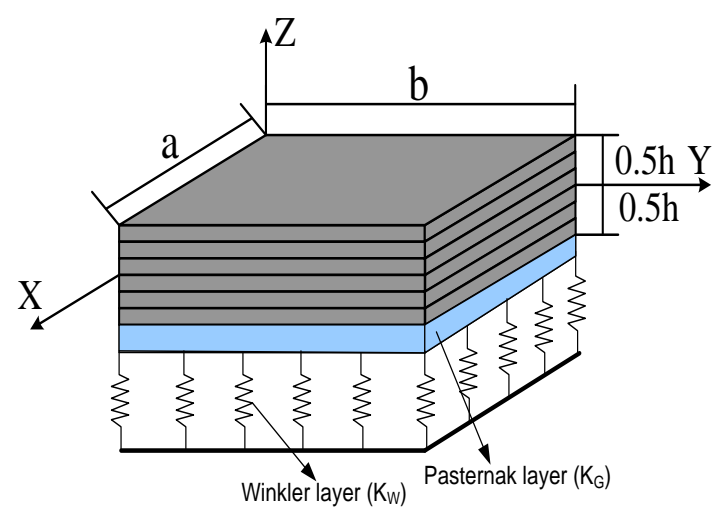

Figure 1. A FG multilayer GPLRC plate on Pasternak foundation model. 
The three distribution patterns of GPL nanofillers across the plate thickness are shown in Figure 2. In the case of X-GPLRC, the surface layers are GPL rich while this is inversed in $\mathrm{O}$ -
GPLRC where the middle layers are GPL rich. As a special case, the GPL content is the same in each layer in a U-GPLRC plate.
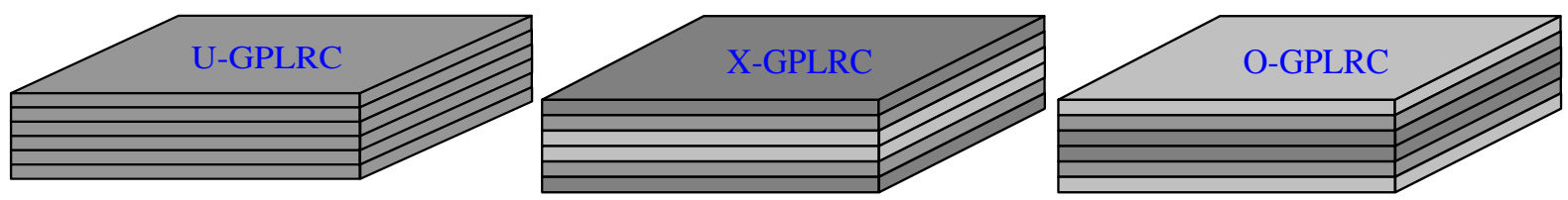

Figure 2. Different GPL distribution patterns in a FG multilayer GPLRC plate.

Functionally graded multilayer GPLRC plates with an even number of layers are considered in this paper. The volume fractions $V_{G P L}$ of the $k$ layer for the three distribution patterns shown in figure 2 are governed by

Case 1: U-

$$
V_{G P L}^{(k)}=V_{G P L}^{*}
$$

GPLRC

GPLRC

$$
V_{G P L}^{(k)}=2 V_{G P L}^{*} \frac{\left|2 k-N_{L}-1\right|}{N_{L}}
$$

Case 3: OGPLRC

$$
V_{G P L}^{(k)}=2 V_{G P L}^{*}\left(1-\frac{\left|2 k-N_{L}-1\right|}{N_{L}}\right)(3)
$$

where $k=1,2,3 \ldots, \mathrm{N}_{L}$ and $N_{L}$ is the total number of layers of the plate. The total volume fraction of GPLs, $V_{G P L}^{*}$, is determined by

$$
V_{G P L}^{*}=\frac{\mathrm{W}_{G P L}}{\mathrm{~W}_{G P L}+\left(\rho_{G P L} / \rho_{m}\right)\left(1-\mathrm{W}_{G P L}\right)}
$$

in which $\mathrm{W}_{G P L}$ is GPL weight fraction; $\rho_{G P L}$ and $\rho_{m}$ are the mass densities of GPLs and the polymer matrix, respectively.

The modified Halpin-Tsai micromechanics model [9] that takes into account the effects of nanofillers' geometry and dimension is used to estimate the effective Young's modulus of GPLRCs

$$
E=\frac{3}{8} \frac{1+\xi_{L} \eta_{L} V_{G P L}}{1-\eta_{L} V_{G P L}} \times E_{m}+\frac{5}{8} \frac{1+\xi_{T} \eta_{T} V_{G P L}}{1-\eta_{T} V_{G P L}} \times E_{m}
$$

Where

$$
\begin{gathered}
\eta_{L}=\frac{\left(E_{G P L} / E_{m}\right)-1}{\left(E_{G P L} / E_{m}\right)+\xi_{L}}, \quad \eta_{T}=\frac{\left(E_{G P L} / E_{m}\right)-1}{\left(E_{G P L} / E_{m}\right)+\xi_{T}}(6) \\
\xi_{L}=2\left(a_{G P L} / t_{G P L}\right), \quad \xi_{T}=2\left(b_{G P L} / t_{G P L}\right)
\end{gathered}
$$

According to the rule of mixture, the Poisson's ratio $v$ and thermal expansion coefficient $\alpha$ of GPLRCs are

$$
\begin{aligned}
& v=v_{m} V_{m}+v_{G P L} V_{G P L} \\
& \alpha=\alpha_{m} V_{m}+\alpha_{G P L} V_{G P L}
\end{aligned}
$$

where $V_{m}=1-V_{G P L}$ is the matrix volume fraction.

\section{Theoretical formulations}

\subsection{Governing equations}

Suppose that the FG multilayer GPLRC plate is subjected to mechanical and thermal loads. In the present study, the first order shear deformation theory (FSDT) is used to obtain the equilibrium, compatibility equations.

According to the FSDT, the displacements of an arbitrary point in the plate are given by [19]

$$
\begin{aligned}
& \bar{U}(X, Y, Z)=U(X, Y)+Z \beta_{X}(X, Y) \\
& \bar{V}(X, Y, Z)=V(X, Y)+Z \beta_{Y}(X, Y) \\
& \bar{W}(X, Y, Z)=W(X, Y)
\end{aligned}
$$

By using von Kármán nonlinearity, the nonlinear strains associated with the displacements are obtained as [19] 


$$
\begin{aligned}
& \left\{\begin{array}{l}
\varepsilon_{X X} \\
\varepsilon_{Y Y} \\
\gamma_{X Y}
\end{array}\right\}=\left\{\begin{array}{c}
\varepsilon_{X}^{0} \\
\varepsilon_{Y}^{0} \\
\gamma_{X Y}^{0}
\end{array}\right\}+z\left\{\begin{array}{c}
\chi_{X} \\
\chi_{Y} \\
\chi_{X Y}
\end{array}\right\}=\left\{\begin{array}{c}
U_{, X}+\frac{1}{2}\left(W_{, X}\right)^{2} \\
V_{, Y}+\frac{1}{2}\left(W_{, Y}\right)^{2} \\
U_{, Y}+V_{, X}+W_{, X} W_{, Y}
\end{array}\right\}+Z\left\{\begin{array}{c}
\beta_{X, X} \\
\beta_{Y, Y} \\
\beta_{X, Y}+\beta_{Y, X}
\end{array}\right\} \\
& \left\{\begin{array}{l}
\gamma_{X Z} \\
\gamma_{Y Z}
\end{array}\right\}=\left\{\begin{array}{l}
W_{, X}+\beta_{X} \\
W_{Y}+\beta_{Y}
\end{array}\right\}
\end{aligned}
$$

where $\varepsilon_{X}^{0}$ and $\varepsilon_{Y}^{0}$ are normal strains and $\gamma_{X Y}^{0}$ is the shear strain in the middle surface of the plate and $\gamma_{X Z}, \gamma_{Y Z}$ are the transverse shear strains components in the plans $X Z$ and $Y Z$ respectively. $U, \quad V, \quad W$ are displacement components corresponding to the coordinates $(\mathrm{X}, \mathrm{Y}, \mathrm{Z}), \beta_{X}$ and $\beta_{Y}$ are the rotation angles of normal vector with $Y$ and $X$ axis.

The stress components of the $k$ layer can be obtained from the linear elastic stress-strain constitutive relationship as

$$
\left\{\begin{array}{l}
\sigma_{X X} \\
\sigma_{Y Y} \\
\sigma_{Y Z} \\
\sigma_{X Z} \\
\sigma_{X Y}
\end{array}\right\}^{(k)}=\left[\begin{array}{ccccc}
B_{11} & B_{12} & 0 & 0 & 0 \\
B_{12} & B_{22} & 0 & 0 & 0 \\
0 & 0 & B_{44} & 0 & 0 \\
0 & 0 & 0 & B_{55} & 0 \\
0 & 0 & 0 & 0 & B_{66}
\end{array}\right]^{(k)}\left\{\left\{\begin{array}{c}
\varepsilon_{X X} \\
\varepsilon_{Y Y} \\
\gamma_{Y Z} \\
\gamma_{X Z} \\
\gamma_{X Y}
\end{array}\right\}^{(k)}-\left\{\begin{array}{c}
\alpha \\
\alpha \\
0 \\
0 \\
0
\end{array}\right\}^{(k)} \Delta T\right)
$$

where $\Delta T$ is the variability of temperature in the environment containing the plate and

$$
B_{11}^{(k)}=B_{22}^{(k)}=\frac{E^{(k)}}{1-v^{2}}, B_{12}^{(k)}=\frac{v E^{(k)}}{1-v^{2}}, B_{44}^{(k)}=B_{55}^{(k)}=B_{66}^{(k)}=\frac{E^{(k)}}{2(1+v)}
$$

According to FSDT, the equations of motion are [19]:

$$
\begin{gathered}
N_{X, X}+N_{X Y, Y}=0, \\
N_{X Y, X}+N_{Y, Y}=0, \\
Q_{X, X}+Q_{Y, Y}+N_{X} W_{, X X}+2 N_{X Y} W_{, X Y}+N_{Y} W_{Y Y}-K_{W} W+K_{G}\left(W_{, X X}+W_{Y Y}\right)=0, \\
M_{X, X}+M_{X Y, Y}-Q_{X}=0, \\
M_{X Y, X}+M_{Y, Y}-Q_{Y}=0,
\end{gathered}
$$

The axial forces $\left(N_{X}, N_{Y}, N_{X Y}\right)$, bending moments $\left(M_{X}, M_{Y}, M_{X Y}\right)$ and shear forces $\left(Q_{X}, Q_{Y}\right)$ are related to strain components by

$$
\left\{\begin{array}{c}
N_{X} \\
N_{Y} \\
N_{X Y}
\end{array}\right\}=[J]\left\{\begin{array}{c}
\varepsilon_{X}^{0} \\
\varepsilon_{Y}^{0} \\
\gamma_{X Y}^{0}
\end{array}\right\}+[C]\left\{\begin{array}{c}
\chi_{X} \\
\chi_{Y} \\
\chi_{X Y}
\end{array}\right\}-\left\{\begin{array}{c}
N^{T} \\
N^{T} \\
0
\end{array}\right\}
$$




$$
\begin{aligned}
& \left\{\begin{array}{c}
M_{X} \\
M_{Y} \\
M_{X Y}
\end{array}\right\}=[C]\left\{\begin{array}{c}
\varepsilon_{X}^{0} \\
\varepsilon_{Y}^{0} \\
\gamma_{X Y}^{0}
\end{array}\right\}+[L]\left\{\begin{array}{c}
\chi_{X} \\
\chi_{Y} \\
\chi_{X Y}
\end{array}\right\}-\left\{\begin{array}{c}
M^{T} \\
M^{T} \\
0
\end{array}\right\} \\
& \left\{\begin{array}{l}
Q_{X} \\
Q_{Y}
\end{array}\right\}=K[P]\left\{\begin{array}{c}
\gamma_{X Z} \\
\gamma_{Y Z}
\end{array}\right\}
\end{aligned}
$$

where shear correction factor $K=5 / 6$. The stiffness elements of the plate are defined as

$$
\begin{aligned}
& \left\{J_{i j}, C_{i j}, L_{i j}\right\}=\sum_{k=1}^{N_{L}} \int_{Z_{k}}^{Z_{k+1}} B_{i j}^{(k)}\left\{1, Z, Z^{2}\right\} d Z,(i, j=1,2,3) \\
& P_{i j}=\sum_{k=1}^{N_{L}} \int_{Z_{k}}^{Z_{k+1}} Q_{i j}^{(k)} d Z,(i, j=1,2),\left\{N^{T}, M^{T}\right\}=\sum_{k=1}^{N_{L}} \int_{Z_{k}}^{Z_{k+1}} Q_{11}^{(k)} \alpha^{(k)} \Delta T\{1, Z\} d Z
\end{aligned}
$$

For using later, the reverse relations are obtained from Eq. (17)

$$
\begin{aligned}
& \varepsilon_{X}^{0}=\frac{J_{12}}{\Delta} N_{Y}-\frac{J_{22}}{\Delta} N_{X}+\frac{J_{22} C_{11}-J_{12} C_{12}}{\Delta} \beta_{X, X}+\frac{C_{12} J_{22}-C_{22} J_{12}}{\Delta} \beta_{Y, Y}+\frac{J_{12}-J_{22}}{\Delta} N^{T} \\
& \varepsilon_{Y}^{0}=N_{X} \frac{J_{12}}{\Delta}-N_{Y} \frac{J_{11}}{\Delta}+\frac{J_{11} C_{22}-C_{12} J_{12}}{\Delta} \beta_{Y, Y}+\frac{C_{12} J_{11}-C_{11} J_{12}}{\Delta} \beta_{X, X}+\frac{J_{12}-J_{11}}{\Delta} N^{T} \\
& \gamma_{X Y}^{0}=\frac{N_{X Y}}{J_{33}}-\frac{C_{33}}{J_{33}} \beta_{X, Y}-\frac{C_{33}}{J_{33}} \beta_{Y, X}
\end{aligned}
$$

where $\Delta=J_{12}^{2}-J_{22} J_{11}$.

The stress function $F(X, Y)$ - the solution of both equations (12) and (13) is introduced as

$$
N_{X}=F_{, Y Y}, N_{Y}=F_{, X X}, N_{X Y}=-F_{, X Y} .
$$

By substituting Eqs. (21), (18) and (19) into Eqs. (14)-(16). Eqs. (14)-(16) can be rewritten

$$
\begin{aligned}
& K P_{44}\left(W_{, X X}+W_{, X X}^{*}\right)+K P_{44} \beta_{X, X}+K P_{55}\left(W_{, Y Y}+W_{, Y Y}^{*}\right)+K P_{55} \beta_{Y, Y} \\
& +F_{, Y Y}\left(W_{, X X}+W_{, X X}^{*}\right)-2 F_{, X Y}\left(W_{, X Y}+W_{, X Y}^{*}\right) \\
& +F_{, X X}\left(W_{, Y Y}+W_{, Y Y}^{*}\right)-K_{W} W+K_{G}\left(W_{, X X}+W_{, Y Y}\right)=0 \\
& S_{21} F_{, X X X}-S_{22} F_{, X Y Y}+S_{23} \beta_{X, X X}+S_{24} \beta_{Y, X Y}+S_{25} \beta_{X, Y Y} \\
& -K P_{44}\left(W_{, X}+W_{, X}^{*}\right)-K P_{44} \beta_{X}=0 \\
& S_{31} F_{, X X Y}+S_{32} F_{, Y Y Y}+S_{33} \beta_{X, X Y}+S_{34} \beta_{Y, X X}+S_{35} \beta_{Y, Y Y} \\
& -K P_{55}\left(W_{, Y}+W_{, Y}^{*}\right)-K P_{55} \beta_{Y}=0
\end{aligned}
$$

where 


$$
\begin{gathered}
S_{21}=\frac{J_{12} C_{11}}{\Delta}-\frac{C_{12} J_{11}}{\Delta}, S_{22}=\frac{J_{22} C_{11}}{\Delta}+\frac{C_{66}}{J_{33}}-\frac{C_{12} J_{12}}{\Delta} \\
S_{23}=\frac{\left(J_{22} C_{11}-J_{12} C_{12}\right) C_{11}}{\Delta}+\frac{\left(C_{12} J_{11}-C_{11} J_{12}\right) C_{12}}{\Delta}+L_{11} \\
S_{24}=\frac{\left(C_{12} J_{22}-C_{22} J_{12}\right) C_{11}}{\Delta}+\frac{\left(J_{11} C_{22}-C_{12} J_{12}\right) C_{12}}{\Delta}+L_{66}+L_{12}-\frac{C_{33} C_{66}}{J_{33}}, S_{25}=L_{66}-\frac{C_{33} C_{66}}{J_{33}} \\
S_{31}=-\frac{C_{66}}{J_{33}}+\frac{J_{12} C_{12}}{\Delta}-\frac{J_{11} C_{22}}{\Delta}, S_{32}=\frac{J_{12} C_{22}}{\Delta}-\frac{J_{22} C_{12}}{\Delta} \\
S_{33}=L_{12}-\frac{C_{33} C_{66}}{J_{33}}+L_{66}+\frac{\left(J_{22} C_{11}-J_{12} C_{12}\right) C_{12}}{\Delta}+\frac{\left(C_{12} J_{11}-C_{11} J_{12}\right) C_{22}}{\Delta} \\
S_{34}=L_{66}-\frac{C_{33} C_{66}}{J_{33}}, S_{35}=\frac{\left(C_{12} J_{22}-C_{22} J_{12}\right) C_{12}}{\Delta}+\frac{\left(J_{11} C_{22}-C_{12} J_{12}\right) C_{22}}{\Delta}+L_{22}
\end{gathered}
$$

The strains are related in the compatibility equation

$$
\varepsilon_{X, Y Y}^{0}+\varepsilon_{Y, X X}^{0}-\gamma_{X Y, X Y}^{0}=\left(W_{, X Y}\right)^{2}-W_{, X X} W_{, Y Y}+2 W_{, X Y} W_{, X Y}^{*}-W_{, X X} W_{, Y Y}^{*}-W_{, Y Y} W_{, X X}^{*}
$$

Set Eqs. (21) and (22) into the deformation compatibility equation (26), we obtain

$$
\begin{aligned}
& -F_{, X X X X} \frac{J_{11}}{\Delta}+\left(\frac{2 J_{12}}{\Delta}+\frac{1}{J_{33}}\right) F_{, X X Y Y}-\frac{J_{22}}{\Delta} F_{, Y Y Y Y}+\left(\frac{J_{22} C_{11}-J_{12} C_{12}}{\Delta}+\frac{C_{33}}{J_{33}}\right) \beta_{X, X Y Y} \\
& +\frac{C_{12} J_{22}-C_{22} J_{12}}{\Delta} \beta_{Y, Y Y Y}+\frac{C_{12} J_{11}-C_{11} J_{12}}{\Delta} \beta_{X, X X X}+\left(\frac{J_{11} C_{22}-C_{12} J_{12}}{\Delta}+\frac{C_{33}}{J_{33}}\right) \beta_{Y, X X Y} \\
& =\left(W_{, X Y}\right)^{2}-W_{, X X} W_{, Y Y}+2 W_{, X Y} W_{, X Y}^{*}-W_{, X X} W_{, Y Y}^{*}-W_{, Y Y} W_{, X X}^{*}
\end{aligned}
$$

The system of fours Eqs. (23) - (25) and (27) combined with boundary conditions and initial conditions can be used for posbuckling of the FG multilayer GPLRC plate.

\subsection{Solution procedure}

Depending on the in-plane behavior at the edges is not able to move or be moved, two boundary conditions, labeled Case 1 and Case 2 will be considered [19]:

Case 1. Four edges of the plate are simply supported and freely movable (FM). The associated boundary conditions are

$$
\begin{aligned}
& W=0, N_{X Y}=0, \beta_{Y}=0, M_{X}=0, N_{X}=N_{X 0} \text { at } X=0, a, \\
& W=0, N_{X Y}=0, \beta_{X}=0, M_{Y}=0, N_{Y}=N_{Y 0} \text { at } Y=0, b .
\end{aligned}
$$

Case 2. Four edges of the plate are simply supported and immovable (IM). In this case, boundary conditions are

$$
\begin{aligned}
& W=U=0, \beta_{Y}=0, M_{X}=0, N_{X}=N_{X 0} \text { at } X=0, a, \\
& \mathrm{~W}=V=0, \beta_{X}=0, M_{Y}=0, N_{Y}=N_{Y 0} \text { at } Y=0, b .
\end{aligned}
$$


where $N_{X 0}, N_{Y 0}$ are the forces acting on the edges of the plate that can be moved (FM), and these forces are the jets when the edges are immovable in the plane of the plate (IM).

The approximate solutions of the system of Eqs. (23)-(25) and (27) satisfying the boundary conditions (28), (29) can be written as

$$
\begin{gathered}
W(\mathrm{X}, \mathrm{Y})=\mathrm{W}_{0} \sin \alpha X \sin \beta Y, \\
\beta_{X}(\mathrm{X}, \mathrm{Y})=\Phi_{X} \cos \alpha X \sin \beta Y, \\
\beta_{Y}(\mathrm{X}, \mathrm{Y})=\Phi_{Y} \sin \alpha X \cos \beta Y, \\
F(\mathrm{X}, \mathrm{Y})=A_{1} \cos 2 \alpha X+A_{2} \cos 2 \beta Y+\frac{1}{2} N_{X 0} Y^{2}+\frac{1}{2} N_{Y 0} X^{2},
\end{gathered}
$$

where $\alpha=\frac{m \pi}{a}, \beta=\frac{n \pi}{b}, m, n=1,2, \ldots$ are the natural numbers of half waves in the corresponding direction $X, Y$, and $W, \Phi_{X}, \Phi_{Y}$ - the amplitudes which are functions dependent on time. The coefficients $A_{i}(i=1-2)$ are determined by substitution of Eqs. $(30,31)$ into Eq. (27) as

$$
A_{1}=f_{1} \mathrm{~W}_{0}^{2}, A_{2}=f_{2} \mathrm{~W}_{0}^{2}, A_{3}=f_{3} \Phi_{x}+f_{4} \Phi_{y}
$$

where

$$
\begin{aligned}
& f_{1}=-\frac{1}{32} \frac{\beta^{2} \Delta}{\alpha^{2} J_{11}} W_{0}\left(W_{0}+\mu h\right), f_{2}=-\frac{1}{32} \frac{\alpha^{2} \Delta}{\beta^{2} J_{22}} W_{0}\left(W_{0}+\mu h\right), \\
& f_{3}=\frac{\alpha^{3} J_{11} J_{33} C_{12}-\alpha^{3} J_{12} J_{33} C_{11}-\alpha \beta^{2} J_{12} J_{33} C_{12}+\alpha \beta^{2} J_{22} J_{33} C_{11}+\Delta \alpha \beta^{2} C_{33}}{\alpha^{4} J_{11} J_{33}-2 \alpha^{2} \beta^{2} J_{12} J_{33}+J_{22} \beta^{4} J_{33}-\Delta \alpha^{2} \beta^{2}} \\
& f_{4}=\frac{\alpha^{2} \beta J_{11} J_{33} C_{22}-\alpha^{2} \beta J_{12} J_{33} C_{12}-\beta^{3} J_{12} J_{33} C_{22}+\beta^{3} J_{22} J_{33} C_{12}+\Delta \alpha^{2} \beta C_{33}}{\alpha^{4} J_{11} J_{33}-2 \alpha^{2} \beta^{2} J_{12} J_{33}+J_{22} \beta^{4} J_{33}-\Delta \alpha^{2} \beta^{2}}
\end{aligned}
$$

Substituting expressions (30)-(32) into Eqs. (23)-(25), and then applying Galerkin method we obtain

$$
\begin{aligned}
& l_{11} W_{0}+3 \pi^{4} m n\left(b^{2} m^{2} N_{x 0}+a^{2} n^{2} N_{y 0}\right)\left(W_{0}+\mu h\right)+l_{12} \Phi_{x}+l_{13} \Phi_{y} \\
& +l_{14}\left(W_{0}+\mu h\right) \Phi_{x}+l_{15}\left(W_{0}+\mu h\right) \Phi_{y}+l_{16} W_{0}\left(W_{0}+\mu h\right)\left(W_{0}+2 \mu h\right)=0 \\
& l_{21} \Phi_{x}+l_{22} \Phi_{y}+l_{23} W_{0}\left(W_{0}+2 \mu h\right)+l_{24}\left(W_{0}+\mu h\right)=0 \\
& l_{31} \Phi_{x}+l_{32} \Phi_{y}+l_{33} W_{0}\left(W_{0}+2 \mu h\right)+l_{34}\left(W_{0}+\mu h\right)=0
\end{aligned}
$$

where

$$
\begin{aligned}
& l_{11}=3 \pi^{4} m n\left(b^{2} m^{2} K_{G}+a^{2} n^{2} K_{G}+a^{2} n^{2} P_{55} K+b^{2} m^{2} P_{44} K\right) \\
& +3 a^{2} b^{2} n \pi^{2} m C_{T}+3 a^{2} b^{2} K_{\mathrm{w}} n \pi^{2} m \\
& l_{12}=3 b^{2} \pi^{3} m^{2} P_{44} K a n, l_{13}=3 a^{2} n^{2} \pi^{3} P_{55} K b m, l_{14}=-32 \pi^{4} f_{3} m^{2} n^{2} \\
& l_{15}=-32 \pi^{4} m^{2} n^{2} f_{4}, l_{16}=6 n^{3} \pi^{6} m^{3}\left(f_{1}+f_{2}\right)
\end{aligned}
$$




$$
\begin{aligned}
& l_{22}=3 b^{2} \pi^{3} m^{3} S_{21} f_{4} n-3 S_{22} m \pi^{3} n^{3} f_{4} a^{2}+3 S_{24} m \pi^{2} n^{2} a^{2} b \\
& l_{21}=3 b^{2} \pi^{3} m^{3} S_{21} f_{3} n-3 S_{22} m \pi^{3} n^{3} f_{3} a^{2}+3 a^{3} \pi^{2} n^{3} S_{25} \\
& l_{23}=-256 S_{21} f_{1} m^{2} \pi b^{2}, l_{24}=3 b^{2} \pi m P_{44} K a^{2} n \\
& l_{31}=3 n \pi^{2} m^{2} S_{33} b^{2} a+3 n \pi^{3} m^{3} S_{31} f_{3} b^{2}+3 a^{2} n^{3} \pi^{3} S_{32} f_{3} m \\
& l_{32}=3 b^{3} \pi^{2} m^{3} S_{34}+3 a^{2} n^{2} \pi^{2} S_{35} m b+3 n \pi^{3} m^{3} S_{31} f_{4} b^{2}+3 a^{2} n^{3} \pi^{3} S_{32} f_{4} m+3 a^{2} b^{3} P_{55} K m, \\
& l_{33}=-256 a^{2} n^{2} \pi S_{32} f_{2}, l_{34}=3 a^{2} n \pi P_{55} K m b^{2}
\end{aligned}
$$

\subsection{Mechanical postbuckling analysis}

Consider the FG multilayer GPLRC plate hinges on four edges which are simply supported and freely movable (corresponding to case 1, all edges FM). Assume that the FG multilayer GPLRC plate is loaded under uniform compressive forces $F_{X}$ and $F_{Y}$ (Pascal) on the edges $X=0$, a, and $Y=0, b$, in which

$$
N_{X 0}=-F_{X} h, N_{Y 0}=-F_{Y} h
$$

Substituting Eq. (36) into Eqs. (33)-(35) leads to the system of differential equations for studying the postbuckling of the plate

$$
\begin{aligned}
& {\left[l_{11}+\frac{\left(l_{22} l_{34}-l_{24} l_{32}\right) l_{12}}{\left(l_{21} l_{32}-l_{22} l_{31}\right)}+\frac{\left(l_{21} l_{34}-l_{24} l_{31}\right) l_{13}}{\left(l_{22} l_{31}-l_{21} l_{32}\right)}\right] \frac{W_{0}}{\left(W_{0}+\mu h\right)}} \\
& +\left[\frac{\left(l_{22} l_{33}-l_{23} l_{32}\right) l_{12}}{\left(l_{21} l_{32}-l_{22} l_{31}\right)}+\frac{\left(l_{21} l_{33}-l_{23} l_{31}\right) l_{13}}{\left(l_{22} l_{31}-l_{21} l_{32}\right)}\right] \frac{W_{0}^{2}}{\left(W_{0}+\mu h\right)}+ \\
& {\left[\frac{\left(l_{22} l_{33}-l_{23} l_{32}\right) l_{14}}{\left(l_{21} l_{32}-l_{22} l_{31}\right)}+\frac{\left(l_{21} l_{33}-l_{23} l_{31}\right) l_{15}}{\left(l_{22} l_{31}-l_{21} l_{32}\right)}\right] W_{0}^{2}+\left[\frac{\left(l_{22} l_{34}-l_{24} l_{32}\right) l_{14}}{\left(l_{21} l_{32}-l_{22} l_{31}\right)}+\frac{\left(l_{21} l_{34}-l_{24} l_{31}\right) l_{15}}{\left(l_{22} l_{31}-l_{21} l_{32}\right)}\right] W_{0}} \\
& +l_{16} W_{0}\left(W_{0}+2 \mu h\right)=3 \pi^{4} m n h\left(b^{2} m^{2} F_{X}+a^{2} n^{2} F_{Y}\right)
\end{aligned}
$$

\subsection{Thermal postbuckling analysis}

Consider the FG multilayer GPLRC plate with all edges which are simply supported and immovable (corresponding to case 2, all edges IM) under thermal load. The condition expressing the immovability on the edges, $\mathrm{U}=0$ (on $\mathrm{X}=0, \mathrm{a}$ ) and $\mathrm{V}=0$ (on $\mathrm{Y}=0, \mathrm{~b}$ ), is satisfied in an average sense as

$$
\int_{0}^{b} \int_{0}^{a} U_{, X} d X d Y=0, \int_{0}^{a} \int_{0}^{b} V_{, Y} d X d Y=0
$$

From Eqs. (9) and (21) of which mentioned relations (22) we obtain the following expressions

$$
\begin{aligned}
& U_{, X}=\frac{J_{12}}{\Delta} N_{Y}-\frac{J_{22}}{\Delta} N_{X}+\frac{J_{22} C_{11}-J_{12} C_{12}}{\Delta} \beta_{X, X}+\frac{C_{12} J_{22}-C_{22} J_{12}}{\Delta} \beta_{Y, Y} \\
& +\frac{J_{12}-J_{22}}{\Delta} N^{T}-\frac{1}{2}\left(W_{, X}\right)^{2} \\
& V_{Y}=N_{X} \frac{J_{12}}{\Delta}-N_{Y} \frac{J_{11}}{\Delta}+\frac{J_{11} C_{22}-C_{12} J_{12}}{\Delta} \beta_{Y, Y}+\frac{C_{12} J_{11}-C_{11} J_{12}}{\Delta} \beta_{X, X} \\
& +\frac{J_{12}-J_{11}}{\Delta} N^{T}-\frac{1}{2}\left(W_{, Y}\right)^{2}
\end{aligned}
$$


Substituting Eqs. (30)-(32) into Eqs. (39), and substituting the expression obtained into Eqs. (38) we have

$$
\begin{gathered}
N_{x 0}=n_{11} \Phi_{X}+n_{12} \Phi_{Y}+n_{13} W_{0}^{2}-N_{T} \\
N_{y 0}=n_{21} \Phi_{X}+n_{22} \Phi_{Y}+n_{23} W_{0}^{2}-N_{T} \\
n_{11}=-\frac{4}{\pi a^{2} b^{2} m n\left(J_{11} J_{22}-J_{12}^{2}\right)}\left(\begin{array}{l}
\left.-J_{11} J_{22} \pi a^{2} n^{2} f_{3}+J_{12}^{2} \pi a^{2} n^{2} f_{3}\right) \\
+J_{11}^{2} C_{11} a b^{2} m-J_{12}^{2} C_{11} a b^{2} m
\end{array}\right) \\
n_{12}=-\frac{4}{\pi a^{2} b^{2} m n\left(J_{11} J_{22}-J_{12}^{2}\right)}\left(\begin{array}{l}
\left.-J_{11} J_{22} \pi a^{2} n^{2} f_{4}+J_{12}^{2} \pi a^{2} n^{2} f_{4}\right) \\
+J_{11} J_{22} C_{12} a^{2} b n-J_{12}^{2} C_{12} a^{2} b n
\end{array}\right) \\
n_{13}=-\frac{\Delta\left(J_{12} a^{2} n^{3} \pi^{3} m+J_{11} \pi^{3} b^{2} m^{3} n\right)}{8 \pi a^{2} b^{2} m n\left(J_{11} J_{22}-J_{12}^{2}\right)} \\
n_{21}=\frac{-4}{\pi a^{2} b^{2} m n\left(J_{11} J_{22}-J_{12}^{2}\right)}\left(\begin{array}{l}
\pi b^{2} J_{12}^{2} m^{2} f_{3}-b^{2} J_{12}^{2} C_{12} a m \\
\left.-J_{22} J_{11} \pi b^{2} m^{2} f_{3}+J_{22} J_{11} C_{12} a b^{2} m\right) \\
n_{22}=\frac{-4}{\pi a^{2} b^{2} m n\left(J_{11} J_{22}-J_{12}^{2}\right)}\left(\begin{array}{l}
-n J_{12}^{2} C_{22} a^{2} b+\pi b^{2} J_{12}^{2} m^{2} f_{4} \\
-J_{22} J_{11} \pi b^{2} m^{2} f_{4}+J_{22} J_{11} C_{22} a^{2} b n
\end{array}\right) \\
n_{23}=-\frac{\left(m^{3} \pi^{3} b^{2} n J_{12}+J_{22} a^{2} n^{3} \pi^{3} m\right) \Delta}{8 \pi a^{2} b^{2} m n\left(J_{11} J_{22}-J_{12}^{2}\right)}
\end{array}\right.
\end{gathered}
$$

Substituting (40) into Eqs (33)-(35) leads to the basic equations used to investigate the postbuckling of the plates in the case all IM edges

where

$$
\begin{aligned}
& p_{1} \frac{W_{0}}{\left(W_{0}+\mu h\right)}+p_{2} W_{0}+p_{3} W_{0}^{2}+p_{4} W_{0}\left(W_{0}+2 \mu h\right) \\
& +p_{5} \frac{W_{0}^{2}}{\left(W_{0}+\mu h\right)}=\left(b^{2} m^{2} 3 \pi^{4} m n+a^{2} n^{2} 3 \pi^{4} m n\right) N_{T}
\end{aligned}
$$

$$
\begin{aligned}
p_{1}= & l_{11}+\frac{\left(l_{22} l_{34}-l_{24} l_{32}\right) l_{12}}{\left(l_{21} l_{32}-l_{22} l_{31}\right)}+\frac{\left(l_{21} l_{34}-l_{24} l_{31}\right) l_{13}}{\left(l_{22} l_{31}-l_{21} l_{32}\right)}, \\
p_{2}= & \frac{\left(l_{22} l_{34}-l_{24} l_{32}\right)\left(n_{21} a^{2} n^{2} 3 \pi^{4} m n+n_{11} b^{2} m^{2} 3 \pi^{4} m n+l_{14}\right)}{\left(l_{21} l_{32}-l_{22} l_{31}\right)} \\
& +\frac{\left(l_{21} l_{34}-l_{24} l_{31}\right)\left(n_{22} a^{2} n^{2} 3 \pi^{4} m n+n_{12} b^{2} m^{2} 3 \pi^{4} m n+l_{15}\right)}{\left(l_{22} l_{31}-l_{21} l_{32}\right)}, \\
p_{3} & =\frac{\left(l_{22} l_{33}-l_{23} l_{32}\right)\left(n_{21} a^{2} n^{2} 3 \pi^{4} m n+n_{11} b^{2} m^{2} 3 \pi^{4} m n+l_{14}\right)}{\left(l_{21} l_{32}-l_{22} l_{31}\right)} \\
& +\frac{\left(l_{21} l_{33}-l_{23} l_{31}\right)\left(n_{22} a^{2} n^{2} 3 \pi^{4} m n+n_{12} b^{2} m^{2} 3 \pi^{4} m n+l_{15}\right)}{\left(l_{22} l_{31}-l_{21} l_{32}\right)}, \\
p_{4} & =\left(n_{23} a^{2} n^{2} 3 \pi^{4} m n+n_{13} b^{2} m^{2} 3 \pi^{4} m n+l_{16}\right), p_{5}=\frac{\left(l_{22} l_{33}-l_{23} l_{32}\right) l_{12}}{\left(l_{21} l_{32}-l_{22} l_{31}\right)}+\frac{\left(l_{21} l_{33}-l_{23} l_{31}\right) l_{13} .}{\left(l_{22} l_{31}-l_{21} l_{32}\right)} .
\end{aligned}
$$




\section{Numerical example and discussion}

The plate $(\mathrm{a} \times \mathrm{b} \times \mathrm{h}=0.45 \mathrm{~m} \times 0,45 \mathrm{~m} \times 0.045 \mathrm{~m})$ is reinforced with GPLs with dimentions $a_{G P L}=2.5 \mu m, b_{G P L}=1.5 \mu m, \mathrm{~h}_{G P L}=15 \mathrm{~nm}$. The material properties of epoxy and GPL are presented in Table 1. In addition, GPL weight fraction is $0.5 \%$ and the total number of layers $N_{L}=10$.

Table 1. Material properties of the epoxy and GPLs [9]

\begin{tabular}{lll}
\hline Material properties & Epoxy & GPL \\
\hline Young's modulus $(\mathrm{GPa})$ & 3.0 & 1010 \\
Density $\left(\mathrm{kg} \cdot \mathrm{m}^{-3}\right)$ & 1200 & 1062.5 \\
Poisson's ratio & 0.34 & 0.186 \\
Thermal expansion coefficient & 60 & 5.0 \\
$\left(\times 10^{-6} / \mathrm{K}\right)$ & & \\
\hline
\end{tabular}

\subsection{Validation of the present formulation}

In table 1, the critical buckling load of FG multilayer GPLRC plate under biaxial compreession $(\mathrm{kN})$ are also compared with those presented in Song et al. [9], in which the authors used a two step perturbation technique [20] to solve differential equations.

According to Table 2, the errors of critical buckling load with Ref. [9] are very small, indicating that the approach of this study is highly reliable.

Table 2. Comparison of critical buckling load of FG multilayer GPLRC plate under biaxial compreession $(\mathrm{kN})$

\begin{tabular}{llcccccc}
\hline & \multicolumn{7}{c}{$\mathrm{W}_{G P L}$} \\
\hline & & Pure epoxy & $0.2 \%$ & $0.4 \%$ & $0.6 \%$ & $0.8 \%$ & $1 \%$ \\
U-GPLRC & Present & 2132.3 & 3547.6 & 4962.3 & 6376.4 & 7789.8 & 9202.7 \\
& Ref. [9] & 2132.3 & 3550.9 & 4968.9 & 6386.3 & 7803.1 & 9219.2 \\
& $\%$ different & 0 & 0.0929 & 0.1328 & 0.155 & 0.1704 & 0.179 \\
& Present & 2132.3 & 4181.8 & 6224.7 & 8265.0 & 10304.0 & 12341.0 \\
X-GPLRC & Ref. [9] & 2132.3 & 4081.3 & 6025.1 & 7966.3 & 9905.7 & 11843.6 \\
& \% different & 0 & 2.462 & 3.313 & 3.75 & 4.021 & 4.2 \\
\hline
\end{tabular}

\subsection{Postbuckling}

Postbuckling curves of the FG multilayer GPLRC plate with different GPL distribution patterns is shown in figures 3 and 4 . It can be seen that the postbucking strength of pattern $\mathrm{X}$ is the best, next is pattern $\mathrm{U}$ and the least pattern $\mathrm{O}$.

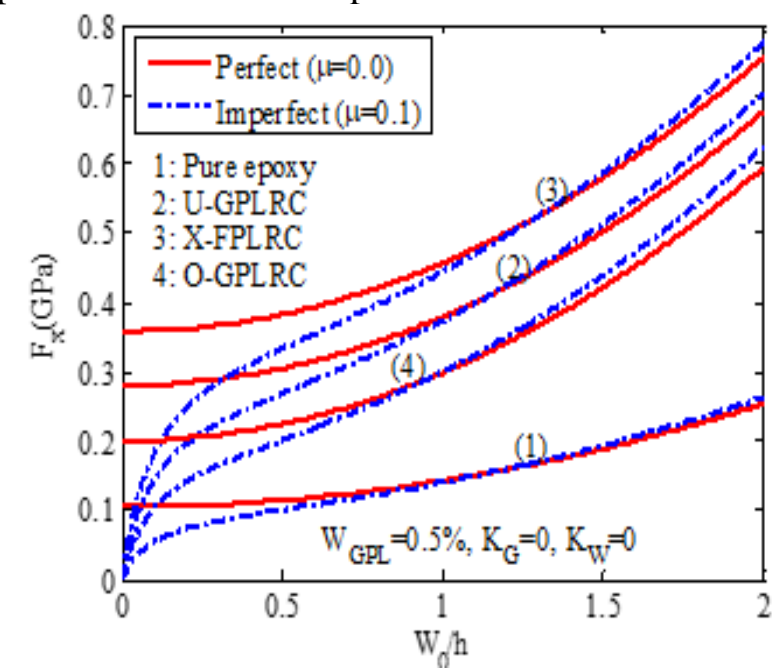

Figure 3. Postbuckling curves of the FG multilayer GPLRC plate under uniaxial compressive load: Effect of GPL distribution pattern.

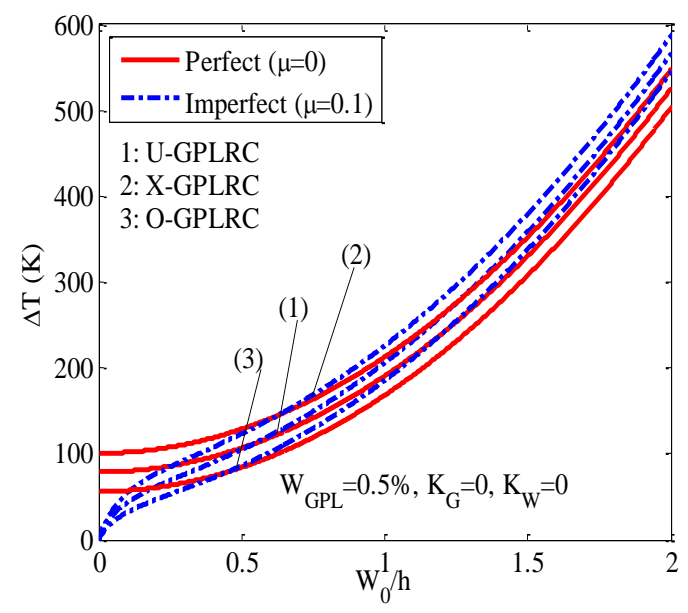

Figure 4. Postbuckling curves of the FG multilayer GPLRC plate under thermal load: Effect of GPL distribution pattern. 


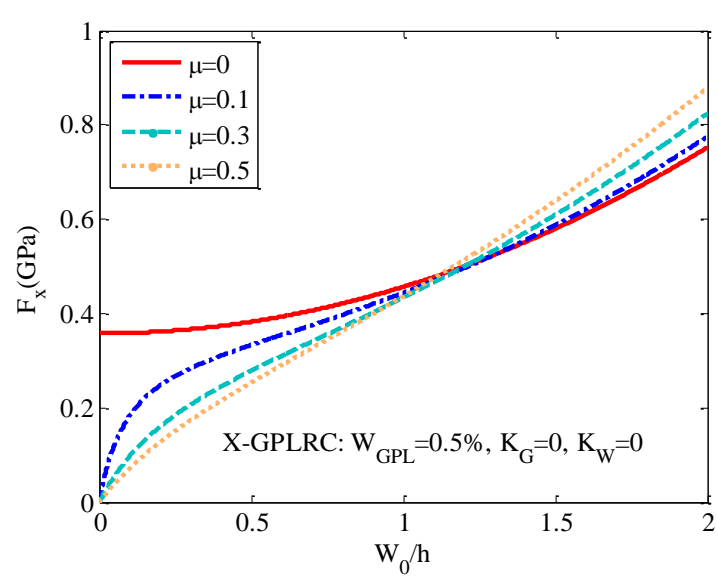

Figure 5. Postbuckling curves of the FG multilayer GPLRC plate under uniaxial compressive load: Effect of imperfection

Figures 5 and 6 show effects of imperfection on buckling and postbuckling curves of the FG multilayer X-GPLRC plate under uniaxial compressive and thermal loads. In postbuckling period, those suggest us that the imperfect properties have affected actively on the loading ability in the limit of large enough $\mathrm{W}_{0} / \mathrm{h}$. In other words, the loading ability increases with $\mu$.

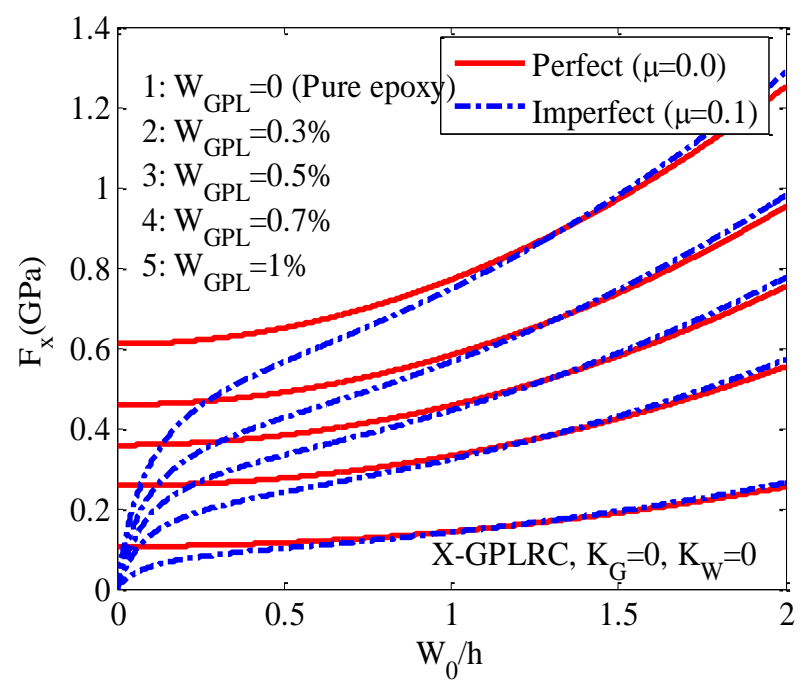

Figure 7. Postbuckling curves of the FG multilayer GPLRC plate under uniaxial compressive load: Effect of GPL weight fraction.

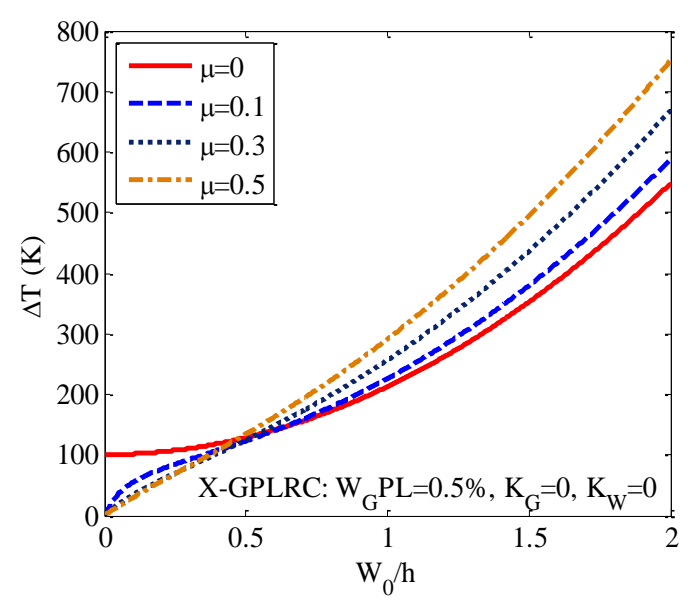

Figure 6. Postbuckling curves of the FG multilayer GPLRC plate under thermal load: Effect of imperfection

Figures 7 and 8 shows the effects of GPL weight fraction $\mathrm{W}_{\mathrm{GPL}}$ on the postbuckling behavior of the FG multilayer X-GPLRC plate under uniaxial compressive and thermal loads. As expected, the postbucking strength of the FG multilayer X-GPLRC plate increased with $\mathrm{W}_{\mathrm{GPL}}$, i.e., with the volume content of GPL in the plate.

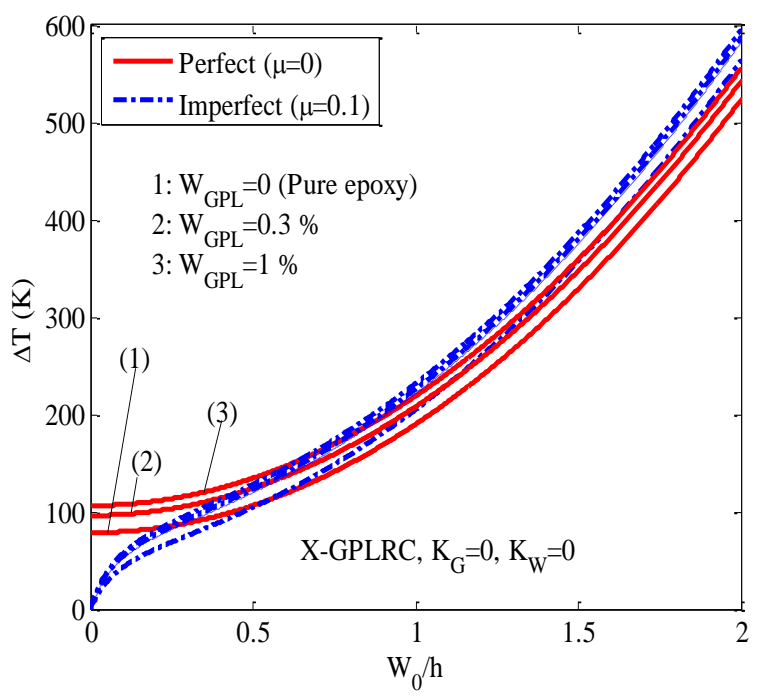

Figure 8. Postbuckling curves of the FG multilayer GPLRC plate under thermal load: Effect of GPL weight fraction. 
Figures 9 and 10 illutrates the effects of GPL width-to thickness ratio $b_{\mathrm{GPL}} / \mathrm{t}_{\mathrm{GPL}}$ and length-towidth ratio $\mathrm{a}_{\mathrm{GPL}} / \mathrm{b}_{\mathrm{GPL}}$ on the postbuckling behavior of the FG multilayer O-GPLRC plates. Figure 9 demonstrates the increased uniaxial compressive postbuckling load - carrying capability of FG multilayer O-GPLRC plates when $b_{\mathrm{GPL}} / \mathrm{t}_{\mathrm{GPL}}$ increases. Figure 10 presents the decreased uniaxial compressive postbuckling load-carrying capability of FG multilayer OGPLRC plates when $\mathrm{a}_{\mathrm{GPL}} / \mathrm{b}_{\mathrm{GPL}}$ increases.

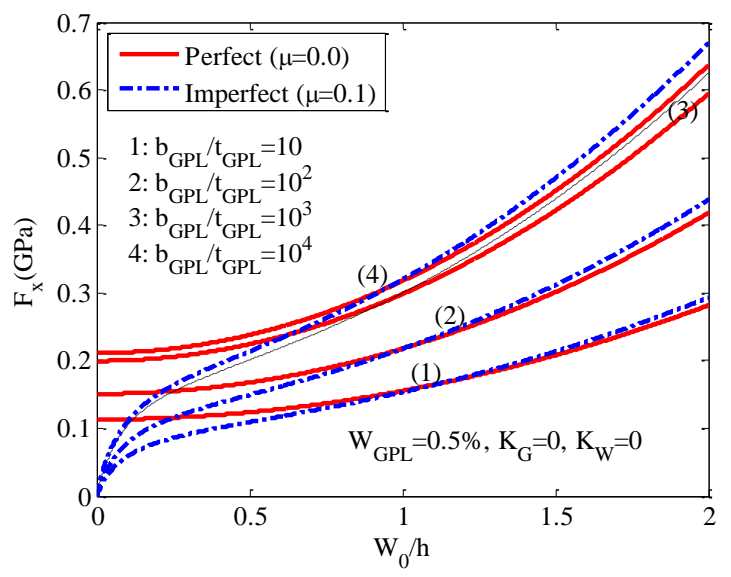

Figure 9. Postbuckling curves of the FG multilayer GPLRC plate under uniaxial compressive load: Effect of GPL length-tothickness ratio.

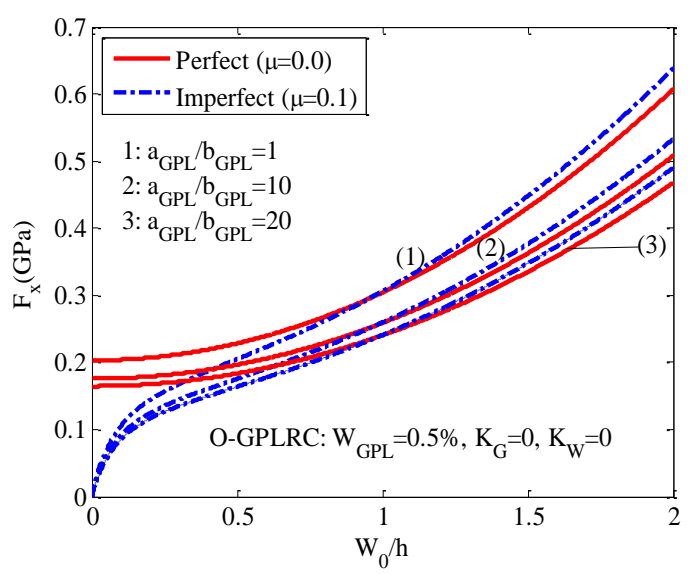

Figure 10. Postbuckling curves of the FG multilayer GPLRC plate under uniaxial compressive load: Effect of GPL length-to-width ratio.

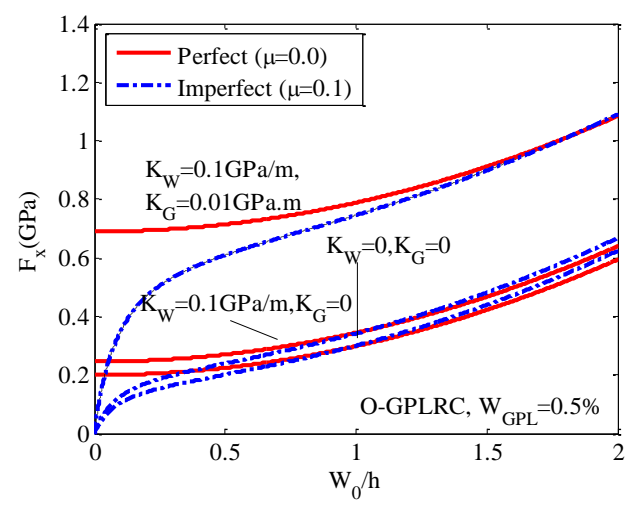

Figure 11. Postbuckling curves of the FG multilayer GPLRC plate under uniaxial compressive load: Effect of elastic foundations.

Figure 11 shows the effects of the elastic foundations on the postbuckling behavior of FG multilayer GPLRC plate. Elastic foundations are recognized to have strong impact, as demonstrated by curve $\left(\mathrm{K}_{\mathrm{W}}=0, \mathrm{~K}_{\mathrm{G}}=0\right)$ and $\left(\mathrm{K}_{\mathrm{W}}=0.1 \mathrm{Gpa} / \mathrm{m}, \mathrm{K}_{\mathrm{G}}=0.01 \mathrm{Gpa} . \mathrm{m}\right)$, which show that the ability of sustaining compression load will increase if the effects of elastic foundations enhance from $\left(K_{W}=0, K_{G}=0\right)$ to $\left(K_{W}=0.1 \mathrm{Gpa} / \mathrm{m}\right.$, $\left.\mathrm{K}_{\mathrm{G}}=0.01 \mathrm{Gpa} . \mathrm{m}\right)$.

\section{Conclusions}

The postbuckling behavior of FG multilayer GPLRC plate under mechanical and thermal loads is investigated based on the FSDT. Some remarkable results are listed following.

- The postbucking strength of pattern $\mathrm{X}$ is the best, next is pattern $\mathrm{U}$ and the least pattern $\mathrm{O}$.

- Elastic foundation models have a positive influence on postbuckling curves, specifically making postbucking strength decrease.

- Increasing the values of GPL weight fraction makes postbucking strength capacity better.

- Effect of geometry and dimension of GPL is also discussed and demonstrated through illustrative numerical examples.

\section{Acknowledgements}

This research is funded by Vietnam National Foundation for Science and Technology Development (NAFOSTED) under grant number 107.02-2018.04. The authors are grateful for this support. 


\section{References}

[1] K.S. Novoselov, A.K. Geim, S.V. Morozov, D. Jiang, Y. Zhang, S.V. Dubonos, I.V. Grigorieva, A. Firsov, Electric filed effect in atomically thin carbon films, Science 306 (2004) 666-669. http://doi.org/ 10.1126/science.1102896.

[2] K.S. Novoselov, D. Jiang, F. Schedin, T.J. Booth, V.V. Khotkevich, S.V. Morozov, A.K. Geim, Two-dimensional atomic crystals, Proceedings of the National Academy of Sciences of the United States of America 102 (2005) 10451-10453. https://doi.org/10.1073/pnas.0502848102.

[3] C.D. Reddy, S. Rajendran, K.M. Liew, Equilibrium configuration and continuum elastic properties of finite sized graphene, Nanotechnology 17 (2006) 864-870. https://doi. org/10.1088/0957-4484/17/3/042.

[4] C. Lee, X.D. Wei, J.W. Kysar, J. Hone, Measurement of the elastic properties and intrinsic strength of monolayer graphene, Science 321 (2008) 385-388. http://doi.org/10.1126/ science. 1157996.

[5] F. Scarpa, S. Adhikari, A.S. Phani, Effective elastic mechanical properties of single layer graphene sheets, Nanotechnology 20 (2009) 065709. https://doi.org/10.1088/0957-4484/20/6/ 065709.

[6] Y.X. Xu, W.J. Hong, H. Bai, C. Li, G.Q. Shi, Strong and ductile poly(vinylalcohol)/graphene oxide composite films with a layered structure, Carbon 47 (2009) 3538-3543. https://doi.org/ 10.1016/j.carbon.2009.08.022.

[7] J.R. Potts, D.R. Dreyer, C.W. Bielawski, R.S. Ruoff, Graphene-based polymer nanocomposites, Polymer 52 (2011) 5-25. https://doi.org/10.1016/j .polymer.2010.11.042.

[8] T.K. Das, S. Prusty, Graphene-based polymer composites and their applications, PolymerPlastics Technology and Engineering 52 (2013) 319-331. https://doi.org/10.1080/03602559.2012. 751410.

[9] M. Song, J. Yang, S. Kitipornchai, W. Zhud, Buckling and postbuckling of biaxially compressed functionally graded multilayer graphene nanoplatelet-reinforced polymer composite plates, International Journal of Mechanical Sciences 131-132 (2017) 345-355. https://doi.org/10.1016/j.ijmecsci.2017.07.017.

[10] H.S. Shen, Y. Xiang, F. Lin, D. Hui, Buckling and postbuckling of functionally graded graphenereinforced composite laminated plates in thermal environments, Composites Part B 119 (2017) $67-$ 78. https://doi.org/10.1016/j.compositesb.2017. 03.020.
[11] H. Wu, S. Kitipornchai, J. Yang, Thermal buckling and postbuckling of functionally graded graphene nanocomposite plates, Materials and Design 132 (2017) 430-441. https://doi.org/10. 1016/j.matdes.2017.07.025.

[12] J. Yang, H. Wu, S. Kitipornchai, Buckling and postbuckling of functionally graded multilayer graphene platelet-reinforced composite beams, Composite Structures 161 (2017) 111-118. https://doi.org/10.1016/j.compstruct.2016.11.048.

[13] H.S. Shen, Y. Xiang, Y. Fan, Postbuckling of functionally graded graphene-reinforced composite laminated cylindrical panels under axial compression in thermal environments, International Journal of Mechanical Sciences 135 (2018) 398-409. https://doi.org/10.1016/j.ijme csci.2017.11.031.

[14] M.D. Rasool, B. Kamran, Stability analysis of multifunctional smart sandwich plates with graphene nanocomposite and porous layers, International Journal of Mechanical Sciences 167 (2019) 105283. https://doi.org/10.1016/j.ijmecs ci.2019.105283.

[15] J.J. Mao, W. Zhang, Buckling and post-buckling analyses of functionally graded graphene reinforced piezoelectric plate subjected to electric potential and axial forces, Composite Structures 216 (2019) 392-405. https://doi.org/10.1016/j. compstruct.2019.02.095.

[16] P.H. Cong, N.D. Duc, New approach to investigate nonlinear dynamic response and vibration of functionally graded multilayer graphene nanocomposite plate on viscoelastic Pasternak medium in thermal environment, Acta Mechanica 229 (2018) 651-3670. https://doi.org/ 10.1007/s00707-018-2178-3.

[17] N.D. Duc, N.D. Lam, T.Q. Quan, P.M. Quang, N.V. Quyen, Nonlinear post-buckling and vibration of 2D penta-graphene composite plates, Acta Mechanica, 2019, https://doi.org/10. 1007/s00707-019-02546-0.

[18] N.D. Duc, P.T. Lam, N.V. Quyen, V.D. Quang, Nonlinear Dynamic Response and Vibration of 2D Penta-graphene Composite Plates Resting on Elastic Foundation in Thermal Environments, VNU Journal of Science: Mathematics-Physics 35(3) (2019) 13-29. https:// doi.org/10.25073/2588-1124/vnumap. 4371.

[19] J.N. Reddy, Mechanics of laminated composite plates and shells; theory and analysis, Boca Raton: CRC Press, 2004.

[20] H.S. Shen, A two-step perturbation method in nonlinear analysis of beams, plates and shells, John Wiley \& Sons Inc., 2013. 